\title{
NURBS-based kinematic limit analysis of FRP-reinforced masonry walls with out-of-plane loading
}

\author{
Andrea Chiozzi \\ University of Ferrara, Italy \\ andrea.chiozzi@unife.it, bttp://orcid.org/0000-0003-4660-0637 \\ Nicola Grillanda, Gabriele Milani \\ Technical University of Milan, Italy \\ nicola.grillanda@polimi.it, \\ gabriele.milani@polimi.it, http://orcid.org/0000-0001-5462-3420
}

Antonio Tralli

University of Ferrara, Italy

tra@unife.it

\begin{abstract}
A three-dimensional (3D) general upper-bound limit analysis procedure for the determination of the collapse load of out-of-plane loaded masonry walls with Fiber Reinforced Polymer (FRP) reinforcement strips is presented. The geometry of a given FRP reinforced masonry wall of arbitrary shape is represented by its Non-Uniform Rational B-Spline (NURBS) description in the three-dimensional Euclidean space. The NURBS parameter space is partitioned by means of a number of possible fracture lines and the original reinforced wall geometry is subdivided into an initial set of rigid elements, accordingly. An upper-bound limit analysis formulation, accounting for the main characteristics of both masonry material and FRP reinforcement by means of homogenization techniques, is deduced. Internal dissipation is allowed along element edges only and the effect of vertical loads and membrane stresses is considered as well. Numerical experiments show that a good estimate of the load bearing capacity is obtained provided that the initial arrangement of yield lines is adjusted by means of a suitable Genetic Algorithm (GA).
\end{abstract}

KEYWORDS. Limit analysis; Masonry; Masonry walls; FRP strips; NURBS; genetic algorithms.

\section{OPEN ACCESS}

Citation: Chiozzi, A., Grillanda, N., Milani, G., Tralli, A.., NURBS-based kinematic limit analysis of FRP-reinforced masonry walls with out-of-plane loading, Frattura ed Integrità Strutturale, 51 (2020) 9-23.

Received: 20.07 .2019

Accepted: 11.10 .2019

Published: 01.01.2020

Copyright: (C) 2019 This is an open access article under the terms of the CC-BY 4.0, which permits unrestricted use, distribution, and reproduction in any medium, provided the original author and source are credited. 


\section{INTRODUCTION}

$\mathrm{M}$

asonry walls represent the commonest structural element encountered in masonry constructions, largely used both as principal members [1] and non-structural internal partitions and infill panels [2]. A relevant issue in the field of structural analysis of existing buildings is the assessment of the load bearing capacity of out-of-plane loaded masonry walls. In fact, out-of-plane failures are mostly a consequence of horizontal actions, especially seismic loads, against which they generally exhibit poor resistance. Recent earthquakes in Italy (L’Aquila, 2009; Emilia 2012; Lazio 2016), demonstrated that out-of-plane failures stem from the lack of out-of-plane strength, especially for historical constructions [3-5]. For this reason, masonry walls in historical buildings often require external retrofitting interventions, in order to guarantee a sufficient level of out-of-plane strength. Since conventional retrofitting solutions - such as the use of steel plates and reinforced concrete plaster - are often impractical and may add undesirable mass to the existing structure, the use of Fiber Reinforced Polymer (FRP) strips is acquiring good popularity in the scientific and professional community. FRP retrofitting strips are characterized by durability, low invasiveness and good ultimate behavior. However, a limited body of work is available to date, addressing the numerical modeling of masonry walls reinforced with FRP strips [6,7]. On the one hand, finite element analyses have been proposed as a viable tool for the prediction of the load bearing capacity of FRP reinforced masonry walls [8]. However, finite element simulations are time expensive and require skilled users to correctly set the many material parameters required in order to provide reliable results. On the other hand, experimental tests carried out since the 70's on masonry walls with out-of-plane loading, have shown that collapse occurs upon formation of a welldefined pattern of linear cracks [9,10]. This evidence inspired approximated solutions relying on both the fracture line theory [11] and the yield line theory [12,13], which in fact are applications of the upper-bound theorem of limit analysis. Indeed, despite the well-established fact that masonry behaves very differently from a rigid-plastic material, limit analysis is among the most reliable tools for the assessment of the load bearing capacity of masonry walls [14]. After Heyman's assumption that masonry behaves as a no-tension material [15], several Italian scientists, among which we recall Como [14], Di Pasquale [16], Angelillo [17], Del Piero [18], have proved that the load bearing capacity of masonry structures can be estimated within the scheme of classic limit analysis under the principles of the theory of plasticity. Nevertheless, several mechanical features of masonry material are not accounted for by the no-tension model. First, masonry is a heterogeneous material with a nonisotropic behaviour, both in the elastic region and at collapse [19]. Second, masonry tensile strength, even if usually very low, is quite variable and uncertain; moreover, the assumption of infinite compression strength is often too strict. Third, experiments show that friction coefficient $\mu$ for masonry is relatively high [20]. Therefore, a non-associative flow rule must be enforced and, as a consequence, limit analysis theorems cannot be applied anymore [21-23]. For such reasons, a number of computational methods for the limit analysis of out-of-plane loaded masonry walls, even in the presence of FRP reinforcement, not based on the no-tension model, have been proposed in literature. For instance, we can recall several Finite Element methods (FEM) based on homogenized limit analysis [24,25].

In the present paper, we propose a new NURBS-based adaptive scheme for the homogenized kinematic limit analysis of masonry walls with out of plane loading, in the presence of FRP reinforcement. The approach, originally proposed by the Authors for both unreinforced and reinforced masonry arches and vaults (see [26-34]), allows to easily assess the out-ofplane failure mechanism for an FRP reinforced wall with openings of arbitrary geometry. Homogenization concepts [35] are employed to obtain out-of-plane homogenized failure mechanical parameters. NURBS (i.e. Non-Rational Uniform BiSpline) are the most common class of approximating basis functions employed in the field of 3D free form modeling [36]. An arbitrary FRP reinforced masonry wall can be represented by a NURBS description of its mid-surface, which can be obtained within any commercial free form modeler, and providing information about the structural thickness at each point of the surface. FRP reinforcement strips are modeled as NURBS surfaces as well. The NURBS parameter space is partitioned by means of a number of possible fracture lines and the original reinforced wall geometry is subdivided into an initial set of rigid elements, accordingly. A homogenized upper-bound limit analysis formulation, accounting for the main characteristics of both masonry material and FRP reinforcement, is deduced. Internal dissipation is allowed along element edges only and the effect of vertical loads and membrane stresses is considered as well. Since the discretization makes use of a very limited number of rigid elements, the actual failure mechanism can be found provided that the discretization is suitably adjusted by means of a Genetic Algorithm (GA) with nonstandard optimization tools [37]. In the process, possible delamination at the FRP/masonry interface is accounted for. Even though delamination is a typically brittle phenomenon, and thus, strictly speaking, it limits the applicability of limit analysis theorems, an equivalent ultimate shear strength for $\mathrm{FRP} /$ masonry interface is assumed in the framework of limit analysis, as suggested by the Italian norm regulating of retrofit interventions with FRP materials [38]. 
With respect to existing methods, the proposed procedure exploits the properties of the NURBS representation of a masonry panel in order to build a very coarse even though exact rigid element discretization. In addition, the knowledge of the actual failure mechanism is not required in advance and the problem is governed by a relatively low number of variables thanks to the use of a very coarse discretization. Therefore, a high computational efficiency is attained. Moreover, since NURBS functions are widespread in the field of free form modeling, the proposed method could easily be integrated within existing commercial CAD software packages, which are popular among professional engineers and architects.

The paper is organized as follows: Section 2 treats the NURBS discretization of a generic masonry wall with openings of arbitrary shape. In Section 3, the proposed upper bound limit analysis NURBS-based adaptive formulation is summarized, which allows to compute the collapse load for a set of given failure mechanisms also accounting for the presence of FRP strips. Finally, Section 4 illustrates the proposed approach by means of numerical simulations.

\section{RIGID ELEMENTS DISCRETIZATION}

$\mathrm{A}$ ny arbitrary FRP reinforced masonry wall with openings, can be modeled as a set of NURBS surfaces within any commercial 3D modeling environment. NURBS basis functions stem from B-splines approximating functions, i.e. piecewise polynomial functions defined by a knot vector $\Xi=\left\{\xi_{1}, \xi_{2}, \ldots, \xi_{n+p+1}\right\}$, whose elements, $\xi_{i} \in[0,1]$, are points in a parametric space. Let us denote by $p$ the polynomial order and $n$ the total number of basis functions. The Coxde-Boor recursion formula allows to compute the $i$-th B-spline basis function, $N_{i, p}[36]$. After defining a suitable set of weights $w_{i} \in \mathbb{R}$, the NURBS basis functions, $R_{i, p}$, is given by:

$$
R_{i, p}(\xi)=\frac{N_{i, p}(\xi) w_{i}}{\sum_{i=1}^{n} N_{i, p}(\xi) w_{i}}
$$

Differently from B-spline basis functions, NURBS basis functions allow the exact representation of the geometry of a wide class of curves such as circles, ellipses, and parabolas [36], as well as the surfaces that can be generated by these curves. In analogy to NURBS curves, it is possible to define a NURBS surface of degree $p$ in the $u$-direction and $q$ in the $v$-direction as the three-dimensional parametric surface defined as:

$$
\mathbf{S}(u, v)=\sum_{i=0}^{n} \sum_{j=0}^{m} R_{i, j}(u, v) \mathbf{B}_{i, j}
$$

where $\left\{\mathbf{B}_{i j}\right\}$ is a lattice of control points in the Euclidean 3D space. Again, it is necessary to set a suitable set of weights $\left\{w_{i, j}\right\}$ and two knot vectors in both $u$ and $v$ directions. Most of the commercial three-dimensional surface modelers, such as Rhinoceros ${ }^{\circledR}[39]$, make use of NURBS functions and their properties to generate and manipulate geometrical objects in the three-dimensional space. In the numerical simulations discussed in Section 4, both masonry walls and FRP reinforcement strips have been modeled within Rhinoceros ${ }^{\circledR}$ as NURBS surfaces. The corresponding NURBS mathematical structure have been exported by means of the IGES (Initial Graphics Exchange Specification) standard [40], so that a suitable (rigid) discretization of the FRP reinforced masonry structure can be constructed in MATLAB ${ }^{\circledR}$, by exploitation of the properties of NURBS functions. In the obtained mesh, each element is a NURBS surface itself and is assumed to be rigid and a thickness is assigned to the elements by offsetting the mid-surface of the desired quantity inward and outward with respect to the normal direction. In fact, the obtained discretization is an assembly of rigid blocks. In the simplest cases, assigned a given masonry wall which can be described by a single NURBS surface, the counter-image $I_{w}$ of its NURBS representation in the $2 \mathrm{D}$ parameters space $u-v$ is the square $[0,1] \times[0,1]$. In general, the counter-image domain $I_{w}$ is bounded by NURBS two-dimensional curves, directly defined in the parameters space. The IGES format stores all the information needed to reconstruct the parameters space of a given NURBS surface.

Subdividing the two-dimensional parameters space $u-v$, it is possible to construct a NURBS discretization of a given planar surface representing a masonry wall. More specifically, in the $2 \mathrm{D}$ parameter space it is possible to define a suitable lattice of nodes, which in fact are the counter-image of the nodes of the actual mesh. Suitable curves in the parameters space, 
connecting the nodes define the edges of the elements in the three-dimensional Euclidean space. An appropriate connectivity matrix for the 2D counter-image of the mesh in the parameters space can be assembled.

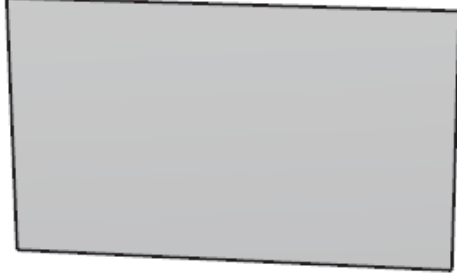

(a)

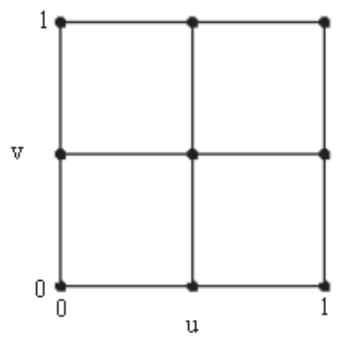

(c)

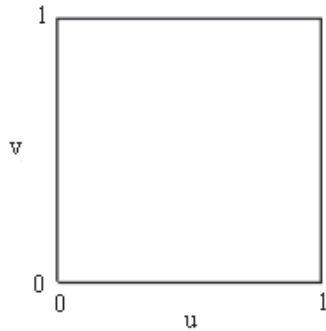

(b)

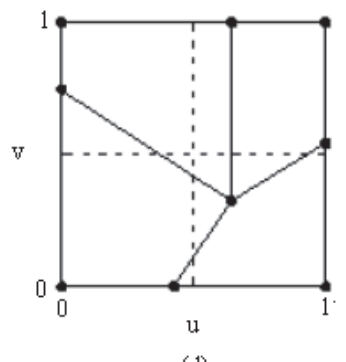

(d)

Figure 1: (a) NURBS model of a masonry wall. (b) Counter-image $I_{\mathrm{w}}$ of the NURBS surface in the parameters space $u-v$. (c) Initial subdivision of the parameters space into four rectangular elements. (d) Modified subdivision of the parameters space into four generic quadrilateral elements.

Fig. 1(a) depicts an example of a 3D NURBS surface used to model a rectangular masonry wall. Fig. 1(b) shows the corresponding parameters space counter-image $I_{w}$, coinciding with the parameters-space $[0,1] \times[0,1]$ itself. Fig. $1(\mathrm{c})$ and Fig. 1(d) depict two possible four-element subdivisions of the parameters space. After the definition of a suitable lattice of nodes in the parameters space, it is possible to transition from the subdivision shown in Fig. 1(c) to the one represented in Fig. 1(d) by controlling the coordinates of the internal nodes. In the proposed approach this process will be suitably automated by means of a GA (see Section 3). Since masonry walls fail upon formation of a limited number of crack lines, the number of nodes belonging to the parameters space lattice can be relatively small [41].

In order to correctly treat openings, a simple algorithm is proposed to generate a robust mesh of the structure. This algorithm is illustrated and summarized in Fig. 2 for a façade wall with two openings containing curved edges. Fig. 2(a) shows the 3D NURBS representation of the wall, while its counter-image $I_{w}$ in the parameters space $[0,1] \times[0,1]$ is depicted in Fig. 2(b). On step one, an initial lattice subdivision of the square $[0,1] \times[0,1]$ in the parameters space is performed, see Fig. 2(c), independently from the presence of openings. Then, the lattice nodes lying outside the actual counter-image of the original surface due to the presence of openings, are moved towards the closest bounding curve of the actual counterimage domain $I_{w}$ of the surface, along rectilinear trajectories parallel to axes $u-v$, as depicted in Fig. 2(d) (step two).

Notable quantities to be computed are area of the surface and the center of mass of each element $E_{i}$ of the mesh. Be $K_{i}$ the counter-image of element $E_{i}$ in the parameters space $u-v$. The surface area of $E_{i}$ can be determined by integration as:

$$
A_{i}=\iint_{E_{i}} d S=\iint_{K_{i}}\left\|\mathbf{S}_{u} \times \mathbf{S}_{\nu}\right\| d u d v
$$

where $\mathbf{S}_{u}$ and $\mathbf{S}_{v}$ are the partial derivatives of surface $\mathbf{S}(u, v)$ along $u$ and $v$ directions. On the other hand, the center of mass of $E_{i}$ may be computed as:

$$
\mathbf{c}=\frac{1}{A_{i}} \iint_{E_{i}} \mathbf{x} d S=\iint_{K_{i}} \mathbf{S}(u, v)\left\|\mathbf{S}_{u} \times \mathbf{S}_{v}\right\| d u d v
$$




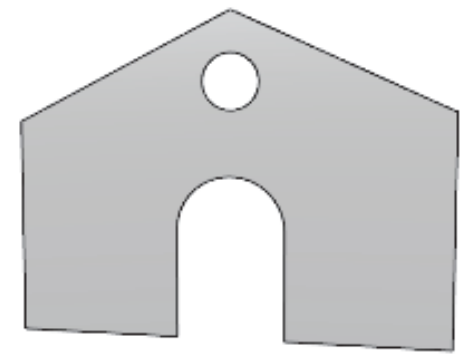

(a)

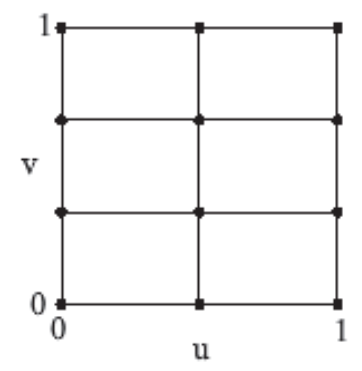

(c)

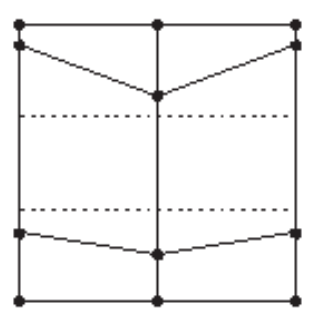

(e)

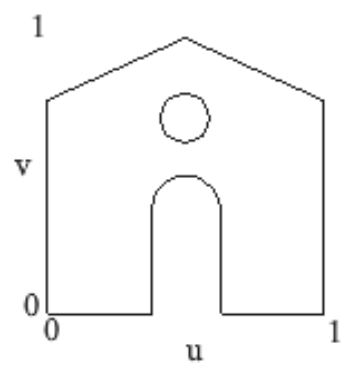

(b)

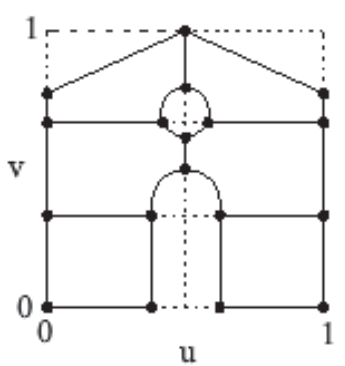

(d)

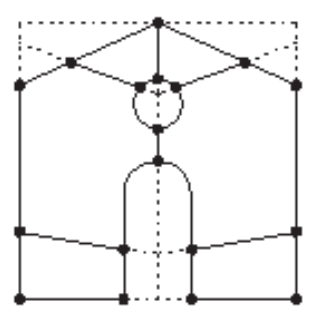

(f)

Figure 2: (a) NURBS model of masonry wall with openings. (b) Counter-image $I_{\mathrm{W}}$ of the NURBS surface in the parameters space $u-v$. (c) Initial subdivision of the parameters space bounding region: step one. (d) Initial subdivision of the counter-image $I_{w}$ of the NURBS surface: step two. (e) GA mesh adaptation in the parameters space bounding region: step one. (f) GA mesh adaptation in the actual parameter space: step two.

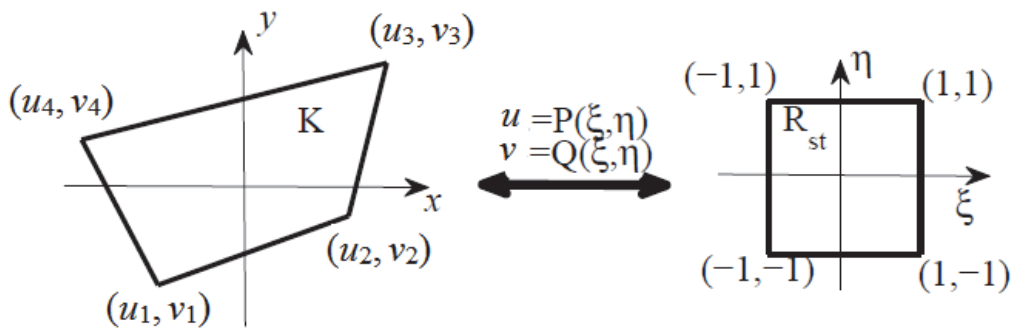

Figure 3: Isoparametric linear mapping.

Integrals (3) and (4) on a generic rectangular domain can be approximated by Gauss numerical cubature coupled with a suitable isoparametric approach, see Fig. 3 and [26] for more details. 


\section{ADAPTIVE NURBS-BASED UPPER-BOUND LIMIT ANALYSIS FORMULATION}

\section{Rigid blocks kinematic limit analysis model}

iven the NURBS rigid blocks discretization model of the FRP reinforced wall, an upper bound limit analysis formulation can be provided, where internal dissipation is allowed only at the element edges. This assumption has proven to be adequate for the general case of FRP reinforced masonry shells with out-of-plane loading [25]. This Section summarizes the proposed limit analysis formulation.

Be $N_{E}$ the number of rigid elements composing the NURBS mesh of the FRP reinforced masonry wall. The kinematics of each element is governed by the six generalized velocities $\left\{u_{x}^{i}, u_{y}^{i}, u_{z}^{i}, \Phi_{x}^{i}, \Phi_{y}^{i}, \Phi_{z}^{i}\right\}$ of its barycenter $G_{i}$. Both dead loads $\mathbf{F}_{0}$ and live loads $\boldsymbol{\Gamma}$ act on the structure. The discretization involves three types of interfaces: masonry-masonry, FRP-masonry and FRP-FRP interfaces. Be $N_{I}^{T O T}=N_{I}^{M-M}+N_{I}^{M-F}+N_{I}^{F-F}$ the number of interfaces, the total internal dissipation rate $D_{\text {int }}$ is given by the sum of the power dissipated along each interface $P_{\text {int }}^{j}$. The total internal dissipation rate $D_{\text {int }}$ is also equal to the sum of the powers of external live $(\mathbf{1} \cdot \Gamma)$ and dead $\left(\mathbf{F}_{0}\right)$ loads, respectively $P_{\Gamma}$ and $P_{\mathbf{F}_{0}}$ :

$$
D_{\mathrm{int}}=\sum_{j=1}^{N_{t}} P_{\mathrm{int}}^{j}=P_{\Gamma}+P_{\mathrm{F}_{0}}
$$

$\Gamma$ is the multiplier of live loads. The Linear Programming (LP) problem associated to the upper-bound formulation of limit analysis requires the minimization of $\Gamma$ under suitable constraints. The unknown of the problem are the set of elemental generalized velocities and plastic multipliers at the interfaces.

Geometrical constraints are imposed by prescribing the values of the generalized velocities at nodes belonging to the element free edges. Therefore, the geometric constraints can be expressed in terms of the generalized velocities at the barycenter of the element containing those nodes, in the following equality form (see [26] for more details):

$$
\mathbf{A}_{e q, \text { geom }} \mathbf{X}=\mathbf{b}_{\text {eq, geom }}
$$

where $\mathbf{A}_{\text {eq, geom }}$ is the matrix of geometric constraints and $\mathbf{b}_{\text {eq, geom }}$ the corresponding vector of coefficients.

With the aim of enforcing plastic compatibility along masonry-masonry interfaces, the edges of each interface have been subdivided into a given number $\left(N_{s d}^{M}+1\right)$ of collocation points $P_{k}$ (see Fig. 4), where a local reference system $\left(\mathbf{n}^{M}, \mathbf{s}^{M}, \mathbf{t}^{M}\right.$ ) is defined, in which $\mathbf{n}^{M}$ is the outward unit vector normal to the interface, $\mathbf{s}^{M}$ is the longitudinal tangential unit vector and $\mathbf{t}^{M}$ is the transversal tangential unit vector. On each point $P_{k}$ of a given interface, between two elements $E^{\prime}$ and $E^{\prime \prime}$, a compatibility relation must hold in the form:

$$
\Delta \tilde{\mathbf{u}}=\dot{\lambda} \frac{\partial f}{\partial \sigma}
$$

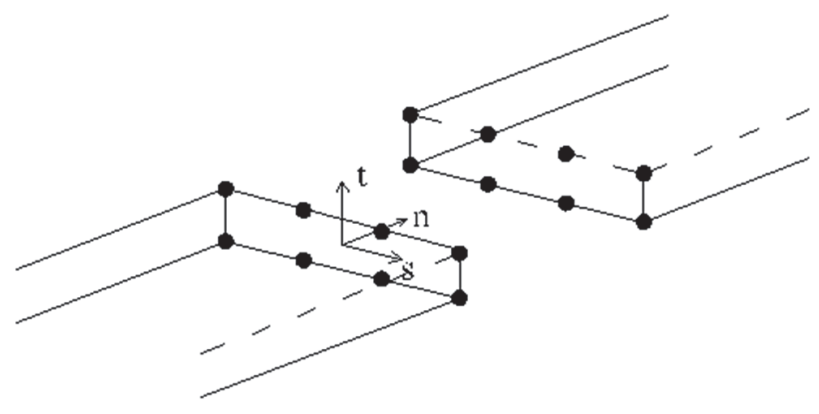

Figure 4: Masonry-masonry interface and local reference system. 
where $\sigma=\left[\sigma_{n n}, \sigma_{n s}, \sigma_{n t}\right]$ is the stress vector in the local reference system at $P_{k}, f(\boldsymbol{\sigma})$ is a suitable yield function and $\dot{\lambda}$ is the (unknown) vector of plastic multipliers. In Eqn. (7), $\Delta \tilde{\mathbf{u}}$ represents the relative velocities at points $P_{k}$ in the local reference system. The same quantity $\Delta \mathbf{u}$ in the global reference system is defined as:

$$
\Delta \mathbf{u}=\mathbf{u}_{P_{i}}^{\prime}-\mathbf{u}_{P_{i}}^{\prime \prime}
$$

being $\mathbf{u}_{P_{i}}^{\prime}$ and $\mathbf{u}_{P_{i}}^{\prime \prime}$ are the velocity vectors at $P_{k}$ on the two sides of the interface. Furthermore, the following relation must hold:

$$
\Delta \tilde{\mathbf{u}}=\tilde{\mathbf{R}} \Delta \mathbf{u}
$$

where $\tilde{\mathbf{R}}$ is a $3 \times 3$ rotation matrix. The yield surface $f(\boldsymbol{\sigma})$ can be obtained by means of a homogenization technique based on the so-called Method of Cells (MoC), first introduced in [42] for unidirectional composites and recently applied to inplane loaded masonry walls [43]. The reader is addressed to [26] for further details. Be the $m$-th plane linearizing $f(\boldsymbol{\sigma})$ defined by the equation $A_{m} \sigma_{n n}+B_{m} \sigma_{n s}+C_{m} \sigma_{n t}=1$. Thus, Eqn. (9) reduces to the equation:

$$
[\Delta \tilde{\mathbf{u}}]_{k}=\left[\begin{array}{c}
\Delta u_{s}^{k} \\
\Delta u_{t}^{k} \\
\Delta u_{n}^{k}
\end{array}\right]=\left[\begin{array}{l}
\sum_{m=1}^{N^{p l}} A_{m} \dot{\lambda}_{m}^{M-M} \\
\sum_{m=1}^{N^{p l}} B_{m} \dot{\lambda}_{m}^{M-M} \\
\sum_{m=1}^{N^{\mu l}} C_{m} \dot{\lambda}_{m}^{M-M}
\end{array}\right]
$$

for each collocation point $P_{k}$, where $\dot{\lambda}_{m}^{M-M}$ is the plastic multiplier corresponding to the $m$-th linearizing plane and $N_{P L}^{M-M}$ is the total number of linearization planes. On each interface $j$, internal dissipation rate in the local reference system reads:

$$
P_{\mathrm{int}, j}^{M-M}=\int_{S_{j}} \boldsymbol{\sigma} \cdot \Delta \tilde{\mathbf{u}} d S
$$

FRP NURBS elements are assumed rigid, as well. Therefore, dissipation is allowed along interfaces between adjacent elements only, and is related to longitudinal stresses in the fibers direction. Again, with the aim of imposing plastic compatibility along FRP-FRP interfaces and correctly evaluating the internal dissipation rate, the mid-line of each interface has discretized into an assigned number $\left(N_{s d}^{F}+1\right)$ of collocation points $P_{k}$ (see Fig. 5).

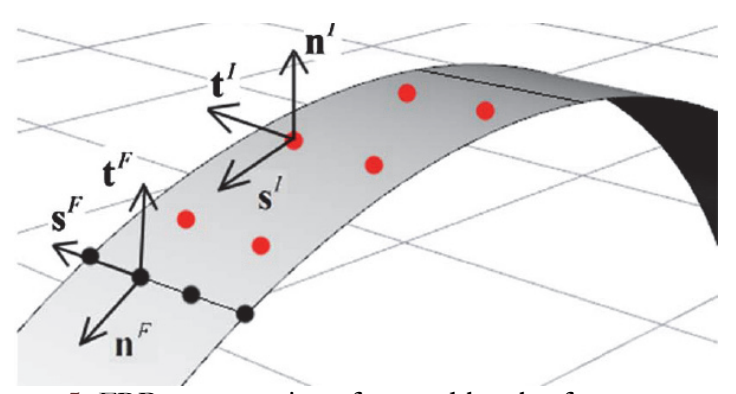

Figure 5: FRP-masonry interface and local reference system.

On each point $P_{k}$, the local reference system $\left(\mathbf{n}^{F}, \mathbf{s}^{F}, \mathbf{t}^{F}\right)$ is introduced, $\mathbf{n}^{F}$ being the outward unit vector normal to the interface, $\mathbf{s}^{F}$ is the longitudinal tangential unit vector and $\mathbf{t}^{F}$ the transversal tangential unit vector. Velocity jumps are 
allowed along direction $\mathbf{n}^{F}$ only. Typically, due to their negligible thickness, FRP strips buckle when subjected to the lowest compression stresses. Therefore, different tension and compression limit stresses are assigned, denoted as $f_{\mathrm{FR} P}^{+}$(equal to $f_{\text {fld }}$ or $f_{\text {fdd, rid }}$ according to [38]) and $f_{F R P}^{-} \approx 0$ respectively. The internal dissipation rate at the $j$-th FRP-FRP interface is computed as:

$$
P_{\mathrm{int}, j}^{F-F}=s \int_{L_{j}} \sigma \cdot \Delta \tilde{\mathbf{u}} d l=s \int_{L_{j}}\left(f_{F R P}^{+} \lambda_{k}^{I-F R P+}+f_{\mathrm{FRP}}^{-} \lambda_{k}^{I-F R P-}\right) d l
$$

where $s$ is the FRP strip thickness, $L_{i}$ the FRP-FRP interface mid-line and $\lambda_{k}^{I-F R P+}$ and $\lambda_{k}^{I-F R P-}$ are plastic multipliers at $P_{k}$ on the interface. Delamination phenomenon depends on a large number of parameters related to materials with very different mechanical behavior (glue, brickwork, fibers). For the sake of simplicity, we adopt the provisions suggested by the Italian technical norm [38]. More precisely, we account for delamination by means of a conventional approach, consisting in suitably limiting the longitudinal tensile stress in the FRP strip - i.e. if the bond length $l_{b}$ is larger than optimal bond length $l_{e}$, the FRP strip design traction strength $f_{\text {fdd }}$ is computed as:

$$
f_{f d d}=\frac{1}{\gamma_{f d} \sqrt{\gamma_{M}}} \sqrt{\frac{2 \cdot E_{F R P} \cdot \Gamma_{F k}}{t_{F R P}}}
$$

whereas if $l_{b} \leq l_{e} f_{f d d}$ is computed as

$$
f_{f d d, r i d}=f_{f d d} \frac{l_{b}}{l_{e}}\left(2-\frac{l_{b}}{l_{e}}\right)
$$

In Eqn. (13) and (14), fffd and $f_{f d d, r i d}$ denote the design bond strength and the reduced design bond strength respectively, while $E_{F R P}$ is the elasticity modulus of FRP, $t_{F R P}$ is the thickness of FRP strip, $\gamma_{f d}$ is a safety factor equal to $1.20, \gamma_{M}$ is the masonry partial safety factor, assumed equal to $1.0, l_{b}$ is the FRP strips bond length and $l_{e}=\sqrt{\frac{E_{F R P} \cdot t_{F R P}}{2 \cdot f_{m t m}}}$ is the optimal bond length. The quantity $\Gamma_{F k}$ in Eqn. (13) represents the specific fracture energy of the FRP reinforced masonry undergoing delamination. A sound estimation of the specific fracture energy is still an open research topic, since mechanical properties of masonry are widely viariable. Nevertheless, as depicted in Fig. 6, the Italian norm prescribes the use of a bilinear $\tau_{b}$-slip constitutive law, allowing the assessment of the limit shear stress $f_{b}$, provided that ultimate slip is known (tipically assumed equal to $0.2 \mathrm{~mm}$ ). As clearly pointed out in many studies, a damaging material model would be the optimal choice when studying failure of FRP strips on masonry $[44,45]$. However, such model cannot be employed in the framework of limit analysis, which, as is well known, relies on the assumption of perfect plasticity. Nevertheless, in agreement with suggestions contained in [38], limit analysis still remains the tool of choice in order to easily estimate the bearing capacity of a given masonry structure. This fact is also reflected in the provisions provided by both the Italian Building Code [46] and the related explicative instructions [47]. Moreover, a number of studies in the literature proved that limit analysis is still capable of reliably assess FRP reinforced masonry structures, see e.g. [48,49].

Finally, FRP-masonry interfaces are NURBS surface belonging to the faces of the masonry wall. To account for dissipation along such third type of interface, a given number $N_{P}^{M-F}$ of collocation points on the interface are defined. At each point we introduce a local reference system $\left(\mathbf{s}^{I}, \mathbf{t}^{I}, \mathbf{n}^{I}\right)$ so that the stress field $\sigma=\left(\tau_{s}, \tau_{t}, \sigma_{n}\right)$ along the local axes is well defined. As before, with the aim of applying the limit analysis theorems, an associate flow rule on the FRP-masonry interface must hold. Again, the failure surface to be used on FRP-masonry interfaces can be linearized as $A_{m} \sigma_{n n}+B_{m} \sigma_{n s}+C_{m} \sigma_{n t}=1$, $m=1, \ldots, N_{P L}^{M-F}$ ( $N_{P L}^{M-F}$ being the number of linearizing planes employed). 


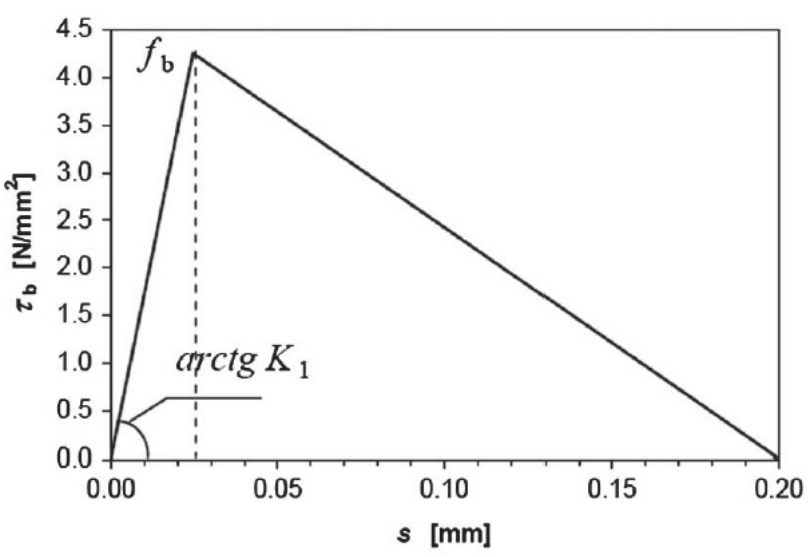

(a)

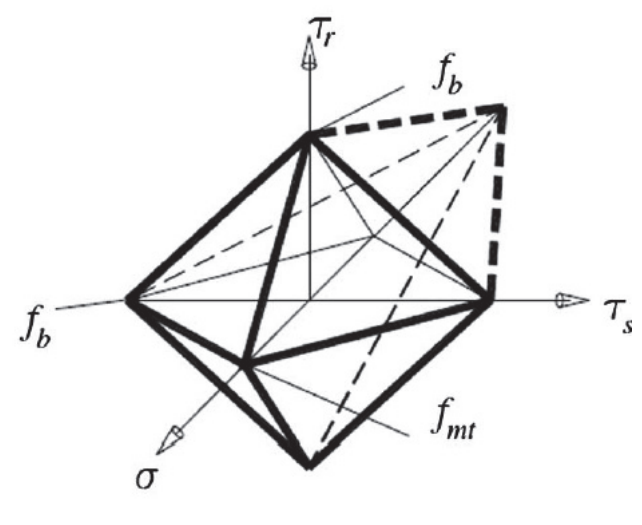

(b)

Figure 6: (a) $\tau_{b}$-slip constitutive law; (b) $\sigma-\tau_{s} \tau_{t}$ failure surfaces for masonry-FRP interfaces.

In this case, flow rule (7) is specialized to:

$$
[\Delta \tilde{\mathbf{u}}]_{k}=\left[\begin{array}{c}
\Delta u_{s}^{k} \\
\Delta u_{t}^{k} \\
\Delta u_{n}^{k}
\end{array}\right]=\left[\begin{array}{l}
\sum_{m=1}^{N_{P L}^{M-F}} A_{m} \dot{\lambda}_{m}^{M-F} \\
\sum_{m=1}^{N_{P-F}^{M-F}} B_{m} \dot{\lambda}_{m}^{M-F} \\
\sum_{m=1}^{M-F} C_{m} \dot{\lambda}_{m}^{M-F}
\end{array}\right]
$$

Eqn. (15) represents further equality constraints to the LP problem, in which $\dot{\lambda}_{m}^{M-F}$ is the $m$-th plastic multiplier associated to the $m$-th linearizing plane. The Italian design code for FRP reinforcement suggest specific $\sigma-\tau_{s}-\tau_{t}$ failure surfaces for FRP-masonry interfaces, as depicted in Fig. 6(b), in which $f_{b}$ is the interface shear strength and $f_{m t}$ describes masonry tensile strength. For each point of each FRP-masonry interface $N_{P L}^{M-F}$ unknown plastic multipliers are introduced. Therefore, the total number of unknown plastic multipliers for FRP-masonry interfaces is equal to $N_{P L}^{M-F} N_{P}^{M-F} N_{I}^{M-F}$. On each FRP-masonry interface $i$, associated to the surface $S_{i}$, the internal dissipation rate is computed in the local reference system as:

$$
P_{\mathrm{int}, j}^{M-F}=\int_{S_{j}} \boldsymbol{\sigma} \cdot \Delta \tilde{\mathbf{u}} d S
$$

Moreover, the non-negativity of each plastic multipliers must be enforced by means of the additional constraint:

$$
\dot{\lambda}_{m} \geq 0 \text {. }
$$

Finally, we must impose a normality condition, requiring that the power dissipated by a unitary live load is equal to one, i.e.:

$$
P_{\Gamma=1}=1
$$

Therefore, the LP problem associated to the proposed upper-bound formulation reads: 


$$
\min \left\{\sum_{j=1}^{N_{I}} P_{\mathrm{int}}^{j}-P_{\mathbf{F}_{\mathbf{0}}}\right\}
$$

under geometric constraints (6), compatibility constraints (10), (12) and (15), non-negativity of plastic multipliers constraints (17) and the normality condition (18).

\section{$G$ A mesh adaptation scheme}

The mesh is adaptively adjusted by means of a GA, allowing the estimation of the minimum collapse multiplier, therefore determining the actual collapse mechanism. A GA is a metaheuristic algorithm for solving both constrained and unconstrained optimization problems, which mimics the process of natural selection and biological evolution [50]. The GA iteratively modifies a population of individual solutions. At each step, individuals are randomly selected from the current population and used as parents to produce next generation offspring, so that the population evolves toward an optimal solution. Here, individuals are a set of parameters defining a mesh and the objective function is the collapse load multiplier.

\section{NUMERICAL EXAMPLES}

$\mathrm{I}$

n this Section we apply the proposed GA-NURBS limit analysis procedure to the study of the ultimate behavior of out-of-plane loaded masonry walls reinforced with FRP strips. The numerical simulations focuses on two FRP reinforced masonry wall specimens subjected to two-way bending with and without openings, fixed at the bottom edge and simply supported along vertical edges. The geometry of the walls and the disposition of FRP reinforcement strips is shown in Fig. 7. In particular, the models reproduce specimens SB01 and SB02 of a set of experimental tests on five unreinforced solid clay bricks masonry panels (labeled from SB01 and SB05) carried out in [51]. All the panels of the experimental campaign have dimensions $5615 \times 2475 \times 102 \mathrm{~mm}$. The panels were loaded in the out-of-plane direction by airbags inflated until failure with increasing pressure $p$. Masonry parameters adopted in the simulations are taken from [51] and [52] (see Tab. 1); no experimental data are available from the literature concerning such panels in presence of FRP reinforcement.

As for the reinforced case, two $100 \mathrm{~mm}$ wide horizontal strips are disposed on the walls extrados, at the top and at the bottom, with the aim of preventing the opening of vertical and inclined cracks observed experimentally in the unreinforced panels. The initial NURBS discretization of the unreinforced panel is made of ten quadrangular elements, obtained by subdividing the parameters space starting from a 4x6 nodes lattice. While the four vertexes are kept fixed, the GA allows to evaluate the optimal position of the remaining fourteen free nodes, minimizing the collapse load multiplier and, thus, obtaining the actual failure mechanism.

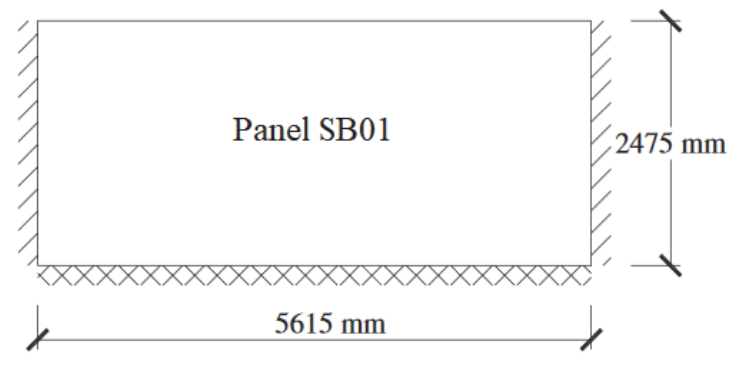

Free edge

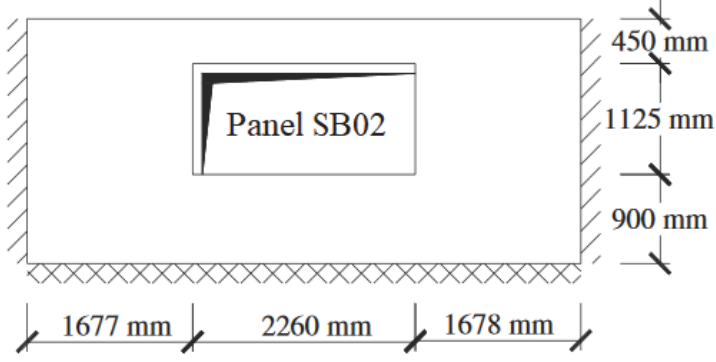

(a)

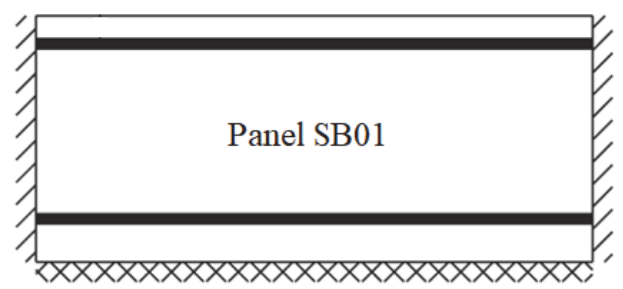

(c)

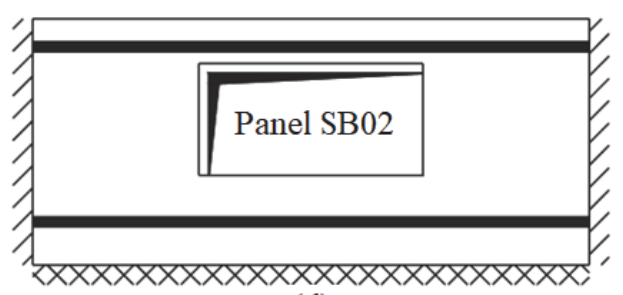

(d)

Figure 7: (a-b) Geometry of wall specimens SB01 and SB02; (c-d) FRP reinforcement configuration for specimens SB01 and SB02. 


\begin{tabular}{ccccc}
\hline Case study & \multicolumn{2}{c}{ Masonry material parameters } & Masonry/FR \\
Compressive strength $\left(\mathrm{f}_{\mathrm{k}}\right)$ & Tensile strength $\left(\mathrm{f}_{\mathrm{t}}\right)$ & $\begin{array}{c}\text { P bond } \\
{[\mathrm{MPa}]}\end{array}$ & $\begin{array}{c}\text { Shear strength } \\
\left(\mathrm{f}_{\mathrm{vk} 0}\right)[\mathrm{MPa}]\end{array}$ & $\begin{array}{c}\text { strength }\left(\mathrm{f}_{\mathrm{b}}\right) \\
{[\mathrm{MPa}]}\end{array}$ \\
$\mathrm{SB} 01, \mathrm{SB} 02[25,51,52]$ & 8.00 & 0.32 & 0.32 & 0.30 \\
\hline
\end{tabular}

Table 1: Material parameters.

Every node position is controlled by two parameters, with the exception of one-parameter edge-nodes and the four fixed vertex-nodes. Thus, also relying on the symmetry of the problem, the optimization problem is governed by fourteen parameters, which can be reduced to nine for to symmetry. A collapse load $p=2.69 \mathrm{kN} / \mathrm{m}^{2}$ has been coputed for the unreinforced case. Fig. 8(a) and Fig. 8(b) respectively depict the computed failure mechanism obtained by means of the proposed GA-NURBS approach and the homogenized FE limit analysis technique proposed in [25]. Moreover, a good agreement can be found when comparing the obtained results from the proposed GA-NURBS approach with the outcomes of both original experiments and different numerical procedures found in the literature [27]. In particular, it can be seen that the proposed GA-NURBS approach slightly overestimates the actual collapse load.

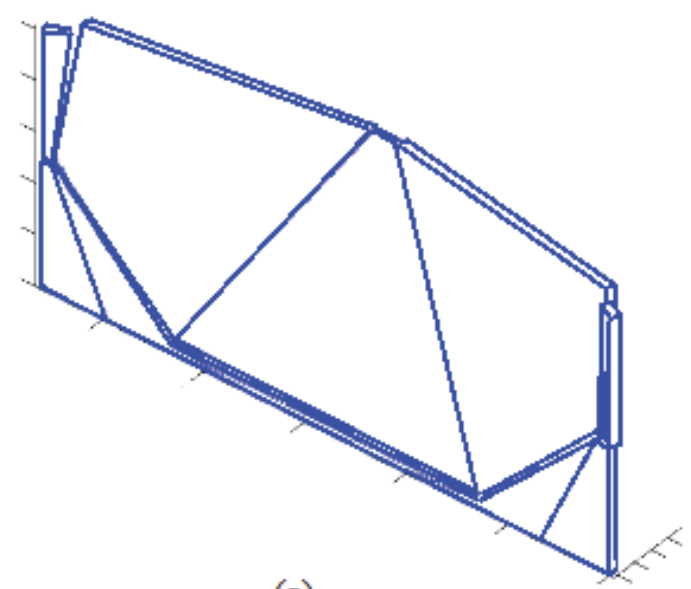

(a)

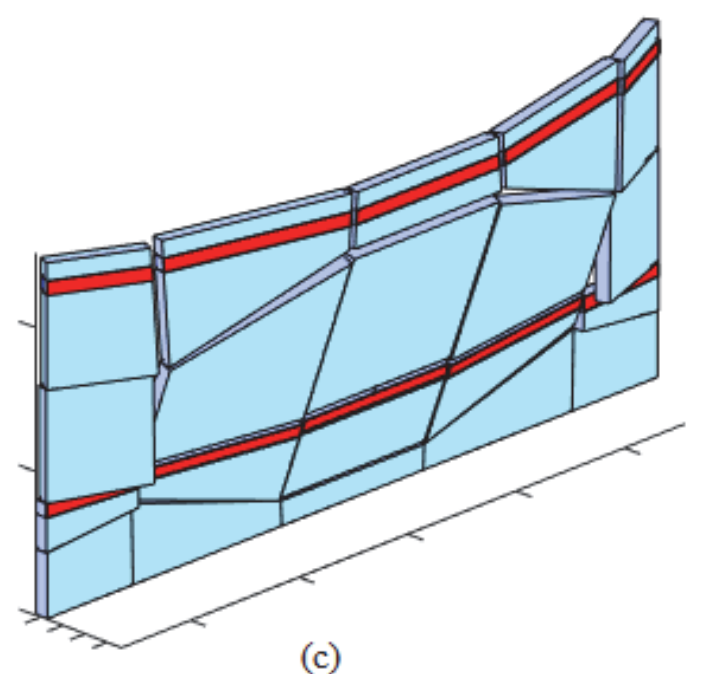

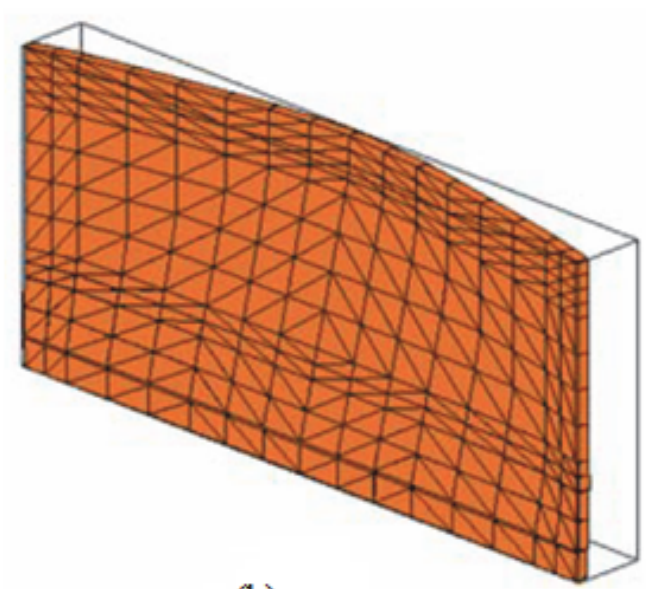

(b)

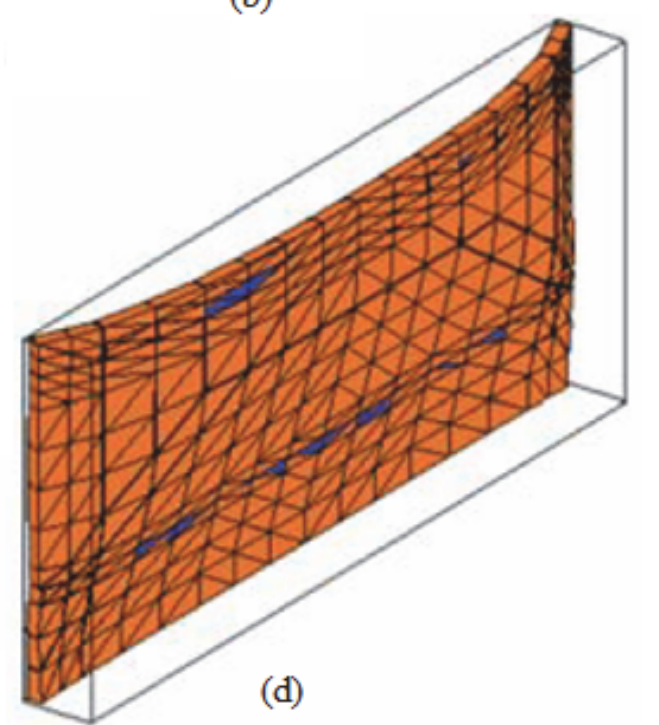

Figure 8: SB01 masonry panel without openings tested in [51]. (a-c) Unreinforced and reinforced case, respectively: collapse mechanism with the GA-NURBS approach; (b-d) Unreinforced and reinforced case, respectively: collapse mechanism with the homogenized FE limit analysis proposed in [25]. 


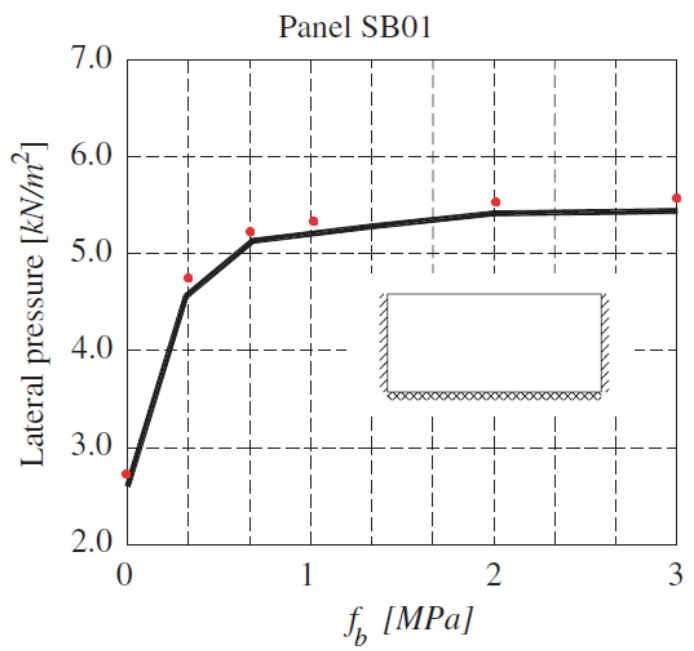

Figure 9: Dependence of the collapse load multiplier of reinforced Panel SB01 on the FRP-masonry bond strength $\mathrm{f}_{\mathrm{b}}$. Red dots: values computed by means of the proposed GA-NURBS approach; Black line: values computed in [25].
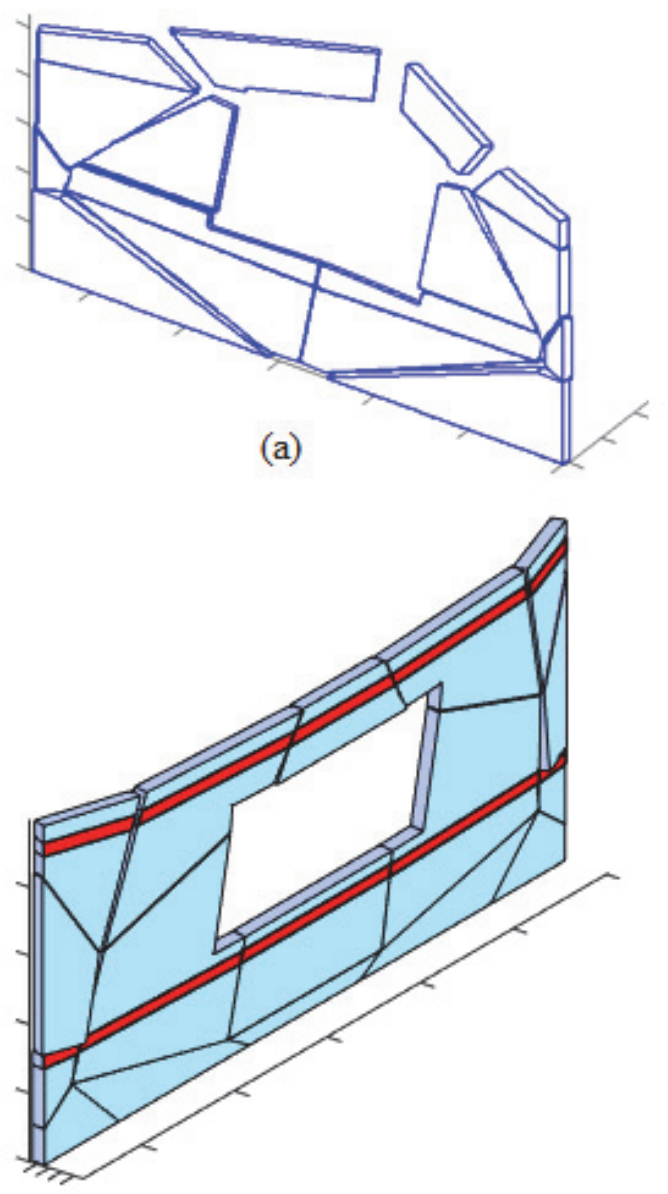

(c)
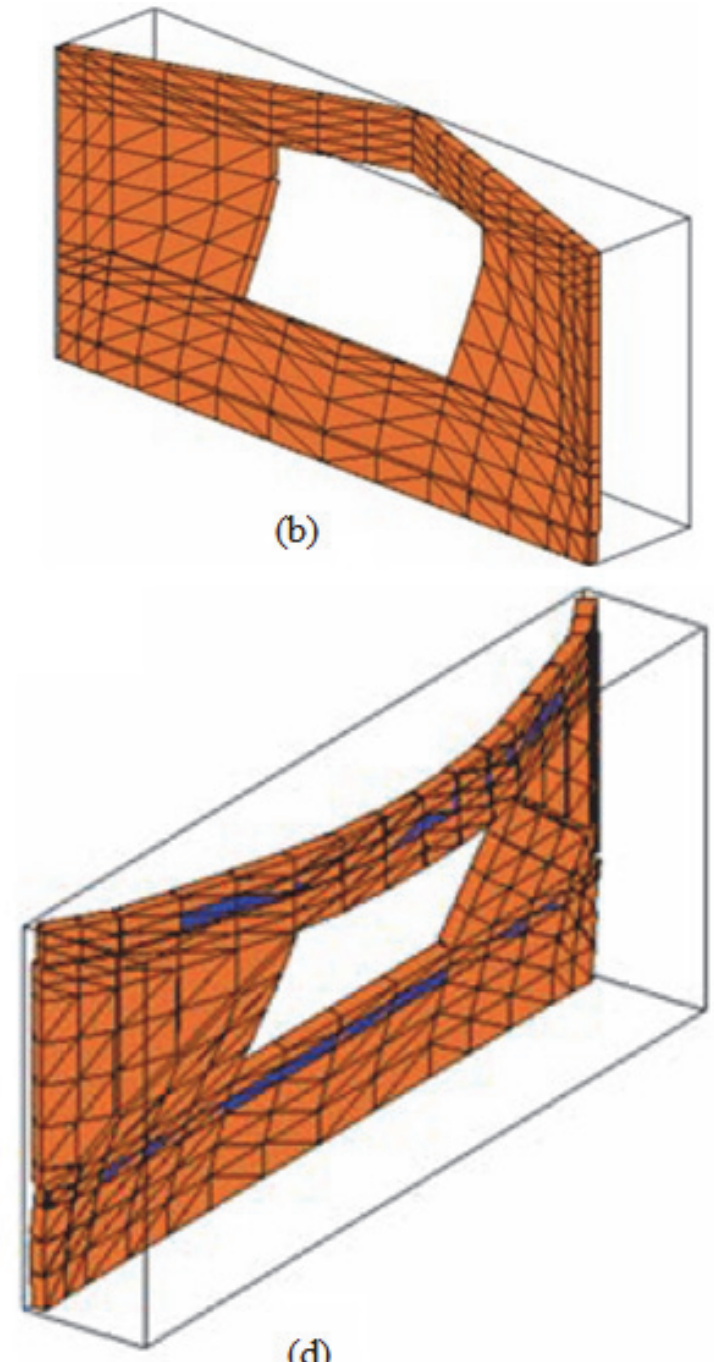

(d)

Figure 10: SB02 masonry panel with rectangular opening tested in [51]. (a-c) Unreinforced and reinforced case, respectively: collapse mechanism with the GA-NURBS approach; (b-d) Unreinforced and reinforced case, respectively: collapse mechanism with the homogenized FE limit analysis proposed in [25]. 
On the other hand, Fig. 8(c) and Fig. 8(d) respectively represent the computed failure mechanism for the reinforced case, obtained by means of the proposed GA-NURBS approach and the homogenized FE limit analysis approach proposed in [25]. A collapse load $p=4.74 \mathrm{kN} / \mathrm{m}^{2}$ has been obtained for the reinforced case, which is in agreement with the collapse load found in [25], i.e. $4.58 \mathrm{kN} / \mathrm{m}^{2}$, as shown in Fig. 9 (for $f_{b}=0.30 \mathrm{MPa}$ ). Fig. 9 also shows the variation in the collapse load multiplier due to a variation in the FRP-masonry bond strength $\mathrm{f}_{\mathrm{b}}$, compared with the values computed in [25].

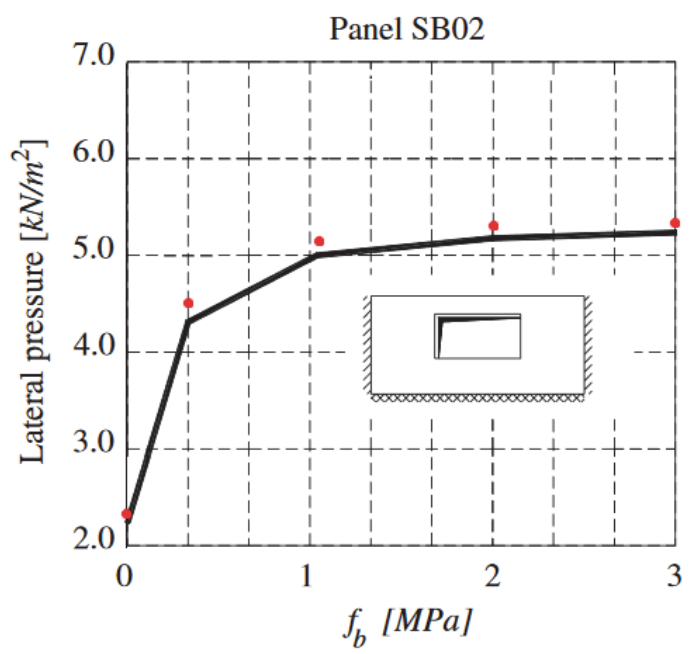

Figure 11: Dependence of the collapse load multiplier of reinforced Panel SB01 on the FRP-masonry bond strength $\mathrm{f}_{\mathrm{b}}$. Red dots: values computed by means of the proposed GA-NURBS approach; Black line: values computed in [25].

Then, the unreinforced panel SB02 with a rectangular opening experimentally tested in [51] is analyzed. The initial NURBS discretization of the wall is made of sixteen quadrangular elements, after subdividing the parameters space from a $4 \times 6$ lattice of nodes. Again the four vertex nodes are fixed. The GA allows to estimate the optimal position of the remaining twentyone nodes, by minimization of the collapse load multiplier, thus obtaining the actual failure mechanism. Every node position is controlled by two parameters, except for the (one parameter) edge-nodes. Finally, appealing to symmetry, the number of governing parameters is reduced to fourteen. A collapse load $p=2.19 \mathrm{kN} / \mathrm{m}^{2}$ has been computed with the proposed GANURBS scheme. Fig. 10(a) and Fig. 10(b) respectively represent the computed failure mechanism obtained through the proposed GA-NURBS approach and the homogenized FE limit analysis approach proposed in [25]. A good agreement is also found between the results from the proposed GA-NURBS approach with the outcomes from both original experiments and different numerical procedures found in the literature [27].

On the other hand, Fig. 10(c) and Fig. 10(d) represent the computed failure mechanism for the reinforced case, computed by means of the proposed GA-NURBS approach and the homogenized FE limit analysis approach proposed in [25], respectively. A collapse $\operatorname{load} p=4.55 \mathrm{kN} / \mathrm{m}^{2}$ has been computed for the reinforced case, which is in agreement with the collapse load found in [25], i.e. $4.30 \mathrm{kN} / \mathrm{m}^{2}$, as shown in Fig. 11 (for $f_{b}=0.30 \mathrm{MPa}$ ). Fig. 11 also shows the dependence of the collapse load multiplier on the FRP-masonry bond strength $\mathrm{f}_{\mathrm{b}}$, compared with the values computed in [25].

\section{CONCLUSIONS}

$\mathrm{W}$ e presented a new GA-NURBS based approach for the upper-bound limit analysis of FRP reinforced masonry walls with out-of-plane loading and arbitrary openings, in which the properties of NURBS functions are exploited to provide an efficient adaptive limit analysis scheme, allowing to easily evaluate both the collapse load multiplier and the failure mechanism of any given FRP reinforced out-of-plane loaded masonry wall, building on its 3D NURBS model. The use of NURBS functions to construct a rigid blocks discretization, allows to easily port the proposed approach into any commercial modeling environment. Moreover, differently from existing procedures implemented in commercial software packages, the GA-NURBS approach does not require an a-priori knowledge of the actual failure mechanism. By means of numerical simulations and comparisons with both experiments and numerical results from the literature, the approach has proved to be capable to accurately predict the ultimate capacity of any FRP reinforced masonry wall with out of plane loading, by using very few elements, and, therefore, maximizing computational speed. 


\section{REFERENCES}

[1] Huerta, S. (2011). The Analysis of Masonry Architecture: A Historical Approach, Archit. Sci. Rev., 51(4), pp. 297-328.

[2] Chiozzi, A., Miranda, E. (2017). Fragility functions for masonry infill walls with in-plane loading, Earthq. Eng. Struct. Dyn., 46(15), pp. 2831-2850, DOI: 10.1002/eqe.2934.

[3] Giuffrè, A. (1993). Safety and Preservation of Historical Centers: the Ortigia case, Laterza (in Italian).

[4] Spence, R., Coburn, A. (1992). Strengthening buildings of stone masonry to resist earthquakes, Meccanica, 27(3), pp. 213-221, DOI: 10.1007/BF00430046.

[5] Chiozzi, A., Simoni, M., Tralli, A. (2016). Base isolation of heavy non-structural monolithic objects at the top of a masonry monumental construction, Mater. Struct. Constr., 49(6), pp. 2113-2130, DOI: 10.1617/s11527-015-0637-z.

[6] Korany, Y., Drysdale, R. (2007). Load-Displacement of Masonry Panels with Unbonded and Intermittently Bonded FRP. II: Analytical Study, J. Compos. Constr., 11(1), pp. 24-32, DOI: 10.1061/(ASCE)1090-0268(2007)11:1(24).

[7] Korany, Y., Drysdale, R. (2007). Load-Displacement of Masonry Panels with Unbonded and Intermittently Bonded FRP. I: Analytical Model, J. Compos. Constr., 11(1), pp. 15-23, DOI: 10.1061/(ASCE)1090-0268(2007)11:1(15).

[8] Monaco, A., Minafò, G., Cucchiara, C., D’Anna, J., La Mendola, L. (2017). Finite element analysis of the out-of-plane behavior of FRP strengthened masonry panels, Compos. Part B Eng., 115, pp. 188-202,

DOI: 10.1016/J.COMPOSITESB.2016.10.016.

[9] De Felice, G., Giannini, R. (2001). Out-of-Plane Seismic Resistance of Masonry Walls, J. Earthq. Eng., 5(2), pp. $253-$ 271.

[10] Gazzola, E.A., Drysdale, R.G., Essawy, A.S. (1985). Bending of concrete masonry walls at different angles to the bed joints, Proc. Third North Amer. Mas. Conf., 27, DOI: 10.1520/STP34544S.

[11] Sinha, B.P. (1978). A Simplified Ultimate Load Analysis of Laterally Loaded Model Orthotropic Brickwork Panels of Low Tensile Strength, Struct. Eng., 56B(4), pp. 81-84.

[12] Losberg, A., Johansson, S. (1969). Sideways pressure on masonry walls of brickwork. CIB Symposium on Bearing Walls, Warsaw, Poland.

[13] Haseltine, B.A., West, H.W.H. (1977). Design of walls to resist lateral loads, Struct. Eng., 55(10), pp. 422-430.

[14] Como, M. (2013). Statics of Historic Masonry Construction, Springer-Verlag Berlin Heidelberg.

[15] Heyman, J. (1966). The stone skeleton, Int. J. Solids Struct., 2(2), pp. 249-79, DOI: 10.1016/0020-7683(66)90018-7.

[16] Di Pasquale, S. (1984). Statica dei solidi murari: teoria ed esperienze, University of Firenze.

[17] Angelillo, M. (1993). Constitutive relations for no-tension materials, Meccanica, 28(3), pp. 195-202, DOI: $10.1007 / \mathrm{BF} 00989121$.

[18] Del Piero, G. (1998). Limit analysis and no-tension materials, Int. J. Plast., 14(1), pp. 259-271, DOI: 10.1016/S0749-6419(97)00055-7.

[19] Dhanasekar, M., Page, A.W., Kleeman, P.W. (1985). The failure of brick masonry under biaxial stresses., Proc. Inst. Civ. Eng., 79(2), pp. 295-313, DOI: 10.1680/iicep.1985.992.

[20] Vasconcelos, G., Lourenço, P.B. (2006).Assessment of the In-plane shear strength of stone masonry walls by simplified models. Proceedings of the 5th International Conference of Structural Analysis of Historical Construction, New Delhi, India.

[21] Radenkovic. (1961). Théorèmes limites pour un materiau de Coulomb a dilatation non standardise, Comptes Rendue Académie Sci. Paris, 252, pp. 4103-4104.

[22] Salençon J. (1977). Application of the theory of plasticity in soil mechanics, John Wiley \& Sons (USA).

[23] Orduña, A., Lourenço, P.B. (2005). Three-dimensional limit analysis of rigid blocks assemblages. Part I: Torsion failure on frictional interfaces and limit analysis formulation, Int. J. Solids Struct., 42(18), pp. 5140-5160,

DOI: 10.1016/j.ijsolstr.2005.02.010.

[24] Grande, E., Milani, G., Sacco, E. (2008). Modelling and analysis of FRP-strengthened masonry panels, Eng. Struct., 30(7), pp. 1842-1860, DOI: 10.1016/j.engstruct.2007.12.007.

[25] Milani, G. (2009). Homogenized limit analysis of FRP-reinforced masonry walls out-of-plane loaded, Comput. Mech., 43(5), pp. 617-639, DOI: 10.1007/s00466-008-0334-7.

[26] Chiozzi, A., Milani, G., Tralli, A. (2017). A Genetic Algorithm NURBS-based new approach for fast kinematic limit analysis of masonry vaults, Comput. Struct., 182, pp. 187-204, DOI: 10.1016/j.compstruc.2016.11.003.

[27] Chiozzi, A., Milani, G., Grillanda, N., Tralli, A. (2017). A fast and general upper-bound limit analysis approach for outof-plane loaded masonry walls, Meccanica, 53(7), pp. 1875-1898, DOI: 10.1007/s11012-017-0637-x.

[28] Chiozzi, A., Milani, G., Tralli, A. (2017). Fast Kinematic Limit Analysis of FRP-Reinforced Masonry Vaults. I: A General 
Genetic Algorithm NURBS-based Formulation, J. Eng. Mech., 143(9), DOI: 10.1061/(ASCE)EM.1943-7889.0001267.

[29] Chiozzi, A., Milani, G., Tralli, A. (2017). Fast kinematic limit analysis of FRP-reinforced masonry vaults. II: Numerical simulations, J. Eng. Mech., 143(9), DOI: 10.1061/(ASCE)EM.1943-7889.0001268.

[30] Chiozzi, A., Grillanda, N., Milani, G., Tralli, A. (2018). UB-ALMANAC: An adaptive limit analysis NURBS-based program for the automatic assessment of partial failure mechanisms in masonry churches, Eng. Fail. Anal., 85, pp. 201220, DOI: 10.1016/j.engfailanal.2017.11.013.

[31] Chiozzi, A., Milani, G., Grillanda, N., Tralli, A. (2016). An Adaptive Procedure for the Limit Analysis of FRP Reinforced Masonry Vaults and Applications, Am. J. Eng. Appl. Sci., 9(3), pp. 735-745, DOI: 10.3844/ajeassp.2016.735.745.

[32] Chiozzi, A., Malagù, M., Tralli, A., Cazzani, A. (2016). ArchNURBS: NURBS-Based Tool for the Structural Safety Assessment of Masonry Arches in MATLAB, J. Comput. Civ. Eng., 30(2), DOI: 10.1061/(ASCE)CP.1943-5487.0000481.

[33] Grillanda, N., Chiozzi, A., Bondi, F., Tralli, A., Manconi, F., Stochino, F., Cazzani, A. (2019). Numerical insights on the structural assessment of historical masonry stellar vaults: the case of Santa Maria del Monte in Cagliari, Contin. Mech. Thermodyn., in press, pp. 1-24, DOI: 10.1007/s00161-019-00752-8.

[34] Grillanda, N., Chiozzi, A., Milani, G., Tralli, A. (2019). Collapse behavior of masonry domes under seismic loads: an adaptive NURBS kinematic limit analysis approach, Eng. Struct., accepted.

[35] Milani, G., Taliercio, A. (2016). Limit analysis of transversally loaded masonry walls using an innovative macroscopic strength criterion, Int. J. Solids Struct., 81, pp. 274-293, DOI: 10.1016/j.ijsolstr.2015.12.004.

[36] Cottrell, J.A., Hughes, T.J.R., Bazilevs, Y. (2009). Isogeometric Analysis: Toward Integration of CAD and FEA, John Wiley \& Sons.

[37] Milani, G., Milani, F. (2008). Genetic algorithm for the optimization of rubber insulated high voltage power cables production lines, Comput. Chem. Eng., 32(12), pp. 3198-3212, DOI: 10.1016/j.compchemeng.2008.05.010.

[38] CNR-DT200. (2013). Instructions for the Design, Execution and Control of retrofit interventions on buildings by means of FRP composites (in italian), Italian Research Council.

[39] McNeel, R. (2008). Rhinoceros: Nurbs Modeling for Windows, Robert McNeel \& Associates.

[40] USPRO. (1996). Initial Graphics Exchange Specification, IGES 5.3, U.S. Product Data Association.

[41] De Felice, G., Universi, R.G., Studi, D., Tre, R. (2001). Out-of-Plane Seismic Resistance of Masonry Walls, J. Earthq. Eng., 5(2), pp. 253-271.

[42] Aboudi, J. (2013). Mechanics of Composite Materials: A Unified Micromechanical Approach, Elsevier.

[43] Milani, G., Taliercio, A. (2015). In-plane failure surfaces for masonry with joints of finite thickness estimated by a Method of Cells-type approach, Comput. Struct., 150, pp. 34-51, DOI: 10.1016/j.compstruc.2014.12.007.

[44] Luciano, R., Sacco, E. (1998). Damage of masonry panels reinforced by FRP sheets, Int. J. Solids Struct., 35(15), pp. 1723-41.

[45] Marfia, S., Sacco, E. (2001). Modeling of reinforced masonry elements, Int. J. Solids Struct., 38(24-25), pp. 4177-4198, DOI: $10.1016 /$ S0020-7683(00)00297-3.

[46] NTC2018. (2018). D.M. 17/01/2018 Aggiornamento delle «Norme tecniche per le costruzioni», S.O. alla G.U. n. 42 del $20 / 02 / 2018$.

[47] CIRC2009. (2009). Instructions for the Application of the New Italian Building Code D.M. 14/01/2008 (in Italian), G.U. n. 47 26/02/2009.

[48] Ascione, L., Feo, L., Fraternali, F. (2005). Load carrying capacity of 2D FRP/strengthened masonry structures, Compos. Part B Eng., 36(8), pp. 619-626, DOI: 10.1016/j.compositesb.2004.12.004.

[49] Caporale, A., Luciano, R., Rosati, L. (2006). Limit analysis of masonry arches with externally bonded FRP reinforcements, Comput. Methods Appl. Mech. Eng., 196(1), pp. 247-260, DOI: 10.1016/j.cma.2006.03.003.

[50] Haupt, R.L., Haupt, S.E. (2004). Practical Genetic Algorithms, John Wiley \& Sons.

[51] Chong, V.L. (1993).The Behavior Of Laterally Loaded Masonry Panels With Openings. University of Plymouth, 1993.

[52] Milani, G., Lourenço, P., Tralli, A. (2006). Homogenization Approach for the Limit Analysis of Out-of-Plane Loaded Masonry Walls, J. Struct. Eng., 132(10), pp. 1650-1663, DOI: 10.1061/(ASCE)0733-9445(2006)132:10(1650). 